Z. vergl. Physiologie 69, 79—98 (1970)

(C) by Springer-Verlag 1970

\title{
Histologische, funktionelle und spezifische Regeneration nach Durchtrennung der Fila olfactoria beim Goldfisch (Carassius auratus)
}

\author{
H. P. Zippex, R. v. Baumgarten, und R. A. Westerman \\ Physiologisches Institut (Lehrstuhl II) und I. Zoologisches Institut der \\ Universität Göttingen
}

Eingegangen am 20. März 1970
Histological, Functional, and Specific Regeneration after Section of Olfactory Fibres in the Goldfish (Carassius auratus)

Summary. 1. After dissection of the olfactory fibres (fila olfactoria, Fig. 1) in the goldfisch, histological (Fig. 5) as well as functional regeneration takes place.

2. When regeneration of the olfactory fibres was complete, no differences in training and learning behaviour between operated and untreated control animals could be observed (Fig. 2).

3. Specific regeneration could also be proved after dissection of the olfactory fibres: Preoperatively trained discriminative behaviour returned (Figs. 3, 4) when histological regeneration had reached an advanced state (Figs. 6, 7).

4. Higher concentrations of all substances used for odour training were capable of exciting the taste receptors or other struktures outside the olfactory mucosa. The concentrations used for olfactory training, however, were below the thresholds for non-olfactory perception.

5. Neither the surgical procedure nor the anesthesia influenced the memory function.

Zusammenfassung. 1. Goldfische können die durchschnittenen Bahnen der Fila olfactoria anatomisch und funktionell regenerieren.

2. Nach Regeneration der Fila olfactoria unterscheiden sich Lernverlauf und Dressurleistungen nicht von intakten Tieren.

3. Nach Durchschneidung der Fila olfactoria konnte eine spezifische Regeneration nachgewiesen werden: Zum Zeitpunkt der fortgeschrittenen histologischen Regeneration stellen sich auch die präoperativ andressierten Verhaltensweisen wieder ein.

4. Alle in der Dressur verwendeten Geruchsstoffe wirken in genügend hoher Konzentration auch als Geschmacksreize (oder Reize auf Rezeptoren außerhalb der Mucosa olfactoria). Die im Geruchstest verwendeten Geruchsstoff-Konzentrationen lagen jedoch unter diesen nicht-olfaktorischen Schwellen. nicht.

5. Die Operation und die Narkose selbst beeinflußten die Gedächnisleistungen

\section{Einleitung}

Die nach zentralnervösen Läsionen einsetzenden Regenerationsprozesse können wir begrifflich dreifach unterteilen: erstens histologische (Wiederbildung der Leitungsstrukturen), zweitens funktionelle (Wieder- 
kehr angeborener Fähigkeiten) und drittens spezifische Regenerationen (Wiederkehr von erworbenen — vor der Läsion erlernten - Differenzierungsleistungen). Im Riechsystem der Fische konnten histologische und funktionelle Regenerationen für den Tractus olfactorius und die Commissura anterior (Zippel und Westerman, 1970, dort weitere Literaturangaben zur Regeneration im ZNS der Fische) nachgewiesen werden.

Neu entwickelte, straffreie Versuchsanlagen zur Geruchsdressur (Zippel, 1970) gestatteten uns, in der vorliegenden Arbeit die Sinnesleistungen der Fische nach Durchtrennung der Fila olfactoria eingehender zu untersuchen. Unser Programm umfaßte: 1. einen Vergleich von operierten Tieren nach Regeneration mit nicht operierten Tieren während der Dressur; 2. die Untersuchung eventueller Geschmackskomponenten der verwendeten Riechstoffe nach Ausschaltung der Geruchsempfindung; 3. die Prüfung der spezifischen Regeneration der Fila olfactoria, durch Testung von voroperativ andressierten Geruchsdifferenzierungsleistungen bei fortschreitender Regeneration, ähnlich wie sie von Sperry et al. im optischen System nachgewiesen wurden.

Histologisch sollte die Regeneration der Fila olf. nach Bulbuskreuzung, d.h. nach Vertauschung der ipsi- und contralateralen Bulbi, vergleichbar mit den Untersuchungen Sperrys (1945) am optischen System, sowie der zeitliche Verlauf der Regeneration der Fila olf. parallel zum Verhaltensexperiment, dargestellt werden.

\section{Methodik}

Die Narkose mit Tricain (Sandoz MS 222) und die Operation mit Eröffnung der Schädelhöhle wurden bereits ausführlich beschrieben (Zippel und Westerman, 1970). Ergänzend sei hier die Durchtrennung der Fila olf. kurz erläutert (Abb. 1).

Die Fila olf. blieben bei der Eröffnung der Schädelhöhle von überhängenden Knochenstückchen verdeckt, die ohne Verletzungen der Nase und damit der

Durchschneidung der:

$\longleftrightarrow$ Fila olfactoria

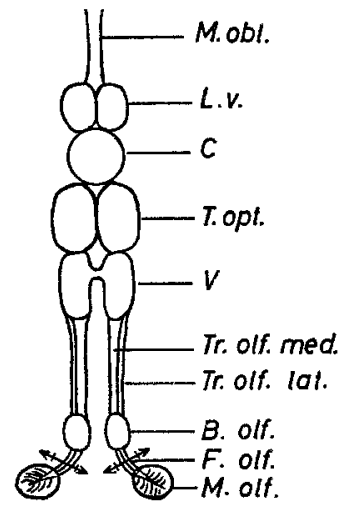

Abb. 1. Schematische Operationsskizze: $M$. obl. Medulla oblongata; $L$. $v$. Lobi vagi; $C$ Cerebellum; T.opt. Tectum opticum; V Vorderhirn; Tr. olf. med. Tractus olfactorius medialis; Tr. oll. lat. Tractus olfactorius lateralis; $B$. olf. Bulbus olfactorius; $F$. olf. Fila olfactoria; $M$. olf. Mucosa olfactoria 
Tabelle 1

\begin{tabular}{|c|c|c|c|c|}
\hline Untersuchung & Dressur & Zahl der Fische & $\begin{array}{l}\text { Bedingter und } \\
\text { unbedingter } \\
\text { Reiz }\end{array}$ & $\begin{array}{l}\text { In der Diffe- } \\
\text { renzdressur } \\
\text { verwendete } \\
\text { andere } \\
\text { Geruchsstoffe }\end{array}$ \\
\hline \multirow{3}{*}{$\begin{array}{l}\text { Funktionelle } \\
\text { Regeneration } \\
\text { der Fila } \\
\text { olfactoria }\end{array}$} & \multirow{3}{*}{$\begin{array}{l}\text { Einzeldressur } \\
\text { postoperativ nach } \\
\text { Wiederherstellung } \\
\text { der Riechfähigkeit }\end{array}$} & 7 (Einzelfische) & $\begin{array}{l}\text { Cumarin } \\
\text { und Tubifex }\end{array}$ & \multirow[t]{2}{*}{ Amylacetat } \\
\hline & & $\begin{array}{l}6 \text { nichtoperierte } \\
\text { Kontrollfische }\end{array}$ & $\begin{array}{l}\text { Cumarin } \\
\text { und Tubifex }\end{array}$ & \\
\hline & & $\begin{array}{l}4 \text { nichtoperierte } \\
\text { Kontrollfische }\end{array}$ & $\begin{array}{l}\text { Amylacetat } \\
\text { und Tubifex }\end{array}$ & Cumarin \\
\hline \multirow[t]{3}{*}{$\begin{array}{l}\text { Spezifische } \\
\text { Regeneration } \\
\text { der Fila } \\
\text { olfactoria }\end{array}$} & $\begin{array}{l}\text { präoperative } \\
\text { Gruppendressur } \\
\text { Operation } \\
\text { fortlaufender Test } \\
\text { ohne Dressur }\end{array}$ & $\begin{array}{l}\text { (2 Gruppen à } 3 \text { ) } \\
\text { I2 } \\
\text { (2 Gruppen à } 3 \text { ) }\end{array}$ & $\begin{array}{l}\text { Cumarin } \\
\text { und Tubifex } \\
\text { Amylacetat } \\
\text { und Tubifex }\end{array}$ & $\begin{array}{l}\text { Cumarin } \\
\text { Amylacetat } \\
\beta \text {-Phenylaethyl- } \\
\text { alkohol } \\
\text { Morpholin } \\
\text { Eugenol } \\
\text { Tubifexextrakt }\end{array}$ \\
\hline & $\begin{array}{l}\text { a) Operation: } \\
\text { Bulbusexstirpation }\end{array}$ & $\begin{array}{l}6 \\
(2 \text { Gruppen à } 3)\end{array}$ & $\begin{array}{l}\text { 1. Gruppe: } \\
\text { Cumarin } \\
\text { und Tubifex } \\
\text { 2. Gruppe: } \\
\text { Amylacetat } \\
\text { und Tubifex }\end{array}$ & \\
\hline & $\begin{array}{l}\text { b) Scheinoperation: } \\
\text { Test }\end{array}$ & $\begin{array}{l}6 \\
(2 \text { Gruppen à } 3 \text { ) }\end{array}$ & $\begin{array}{l}\text { 1. Gruppe: } \\
\text { Cumarin } \\
\text { und Tubifex } \\
\text { 2. Gruppe: } \\
\text { Amylacetat } \\
\text { und Tubifex }\end{array}$ & \\
\hline
\end{tabular}

Nasenschleimhaut nicht entfernt werden konnten. Ein feingezogener, an der Spitze gebogener und stumpfer Glasstab wurde von caudo-lateral hinter den einen Bulbus olfactorius geschoben und dann an der Innenseite des Schädels entlang nach cranio-medial geführt; dabei zerrissen die Fila olfactoria. Der losgelöste Bulbus wurde mit dem. Glasstab vorsichtig um etwas mehr als eine Bulbuslänge nach hinten geschoben, um sicherzustellen, daß alle Fila olfactoria restlos durchtrennt waren. Bei der Bulbuskreuzung wurde darauf geachtet, daß die dorsale und ventrale Seite der Bulbi in unveränderter Orientierung blieben.

\section{Ausarbeitung und Prüfung des bedingten Reflexes}

Auf die Ausschaltung störender Außenfaktoren während der Dressur der Versuchstiere sind wir in einer früheren Arbeit (Zippel und Westerman, 1970) bereits eingegangen. Ebenso wurden die verwendeten Versuchsanlagen sowie die Dressur ein- 
gehend beschrieben (Zippel, 1970). Die Untersuchungen wurden mit verschiedenen, für die Versuchstiere unbekannten Duftstoffen (s. Tabelle 1 und 2) durchgeführt. Die Geruchsstofflösungen strömten in einer Konzentration von $1 \times 10^{-6}$ bis $1 \times 10^{-7}$ $\mathrm{ml}$ konzentrierten Geruchsstoffes pro $1 \mathrm{ml}$ Wasser in die Versuchsbecken ein und vermischten sich dann mit dem Wasser des Aquariums. Als unbedingten Reiz verwendeten wir in allen Fällen Tubifex.

Zur Prüfung der funktionellen Regeneration der Fila olfactoria (Test: s. Zippel und Westerman, 1970) und zu der 14-28 Tage dauernden Gewöhnung an die Versuchsanlage (Trichterfütterung) dressierten wir die operierten parallel mit den nicht operierten Fischen zunächst auf einen Geruchsstoff (Cumarin), anschließend in einer Differenzdressur auf Cumarin als "positiven" und Amylacetat als ", negativen " Riechstoff (Tabelle 1). Jede Dressur war zugleich auch ein Test (5--30 min Test, anschließend Fütterung bei weiterlaufendem Geruchsstoff). Zur Prüfung der spezifischen Regeneration wurden die Tiere nach Erreichen positiver Kriterien in der Differenzdressur operiert (Durchschneidung der Fila olfactoria). Anschließend prüften wir ihre Reaktion gegenüber den Riechstoffen (Tabelle 1) mehrmals täglich. Die in der Dressur im Anschluß an den Test folgende Fütterung mußte dabei unterbleiben.

Die histologische Aufarbeitung der Gehirne mit einer Färbung nach Bodian ist in einer früheren Arbeit (Zippel und Westerman, 1970) beschrieben.

\section{Ergebnisse}

\section{Dressur von Einzelfischen (funktionelle Regeneration)}

Verhaltensunterschiede in der Dressur mit einem Riechstoff zwischen operierten und nicht operierten Fischen müBten in der Verhaltensanalyse deutlich hervortreten. Schon bei oberflächlicher Betrachtung der Schwimmregistrierungen von operierten Fischen (Abb. 2A, B) finden sich aber auffallende Parallelen zu nicht operierten Tieren.

Für die genauere Auswertung der Registrierungen wurden aus jeder Gruppe Fische mit möglichst gleichartigem Verhalten gegenübergestellt. Ein Vergleich der typischen Verhaltensmerkmale in der Dressur ist bei Zippel (1970) dargestellt. In der Schwimmgeschwindigkeit, in der Anzahl der Bisse auf der positiven Seite, in der Zahl der geschwommenen Kreisbewegungen und im Aufenthalt auf der positiven Seite zeigen operierte Tiere nach Regeneration der Fila olfactoria in der Dressur genau das gleiche Verhalten wie nicht-operierte Tiere. Nach 80-100 Dressuren verhalten sich alle Einzelfische deutlich positiv gegenüber dem Geruchsstoff.

In den ersten Differenzdressuren finden wir auch bei operierten Tieren das schon früher (Zippel, 1970) für nicht-operierte Fische beschriebene, stark quantitäts- (konzentrations) abhängige Verhaltengegenüber den gebotenen Riechstoffen. Im weiteren Verlauf der Differenzdressur (Abb. 2C, D) lernen unsere operierten Tiere den positiven Geruchsstoff anhand seiner Qualität unabhängig von der Quantität positiv zu bewerten. 

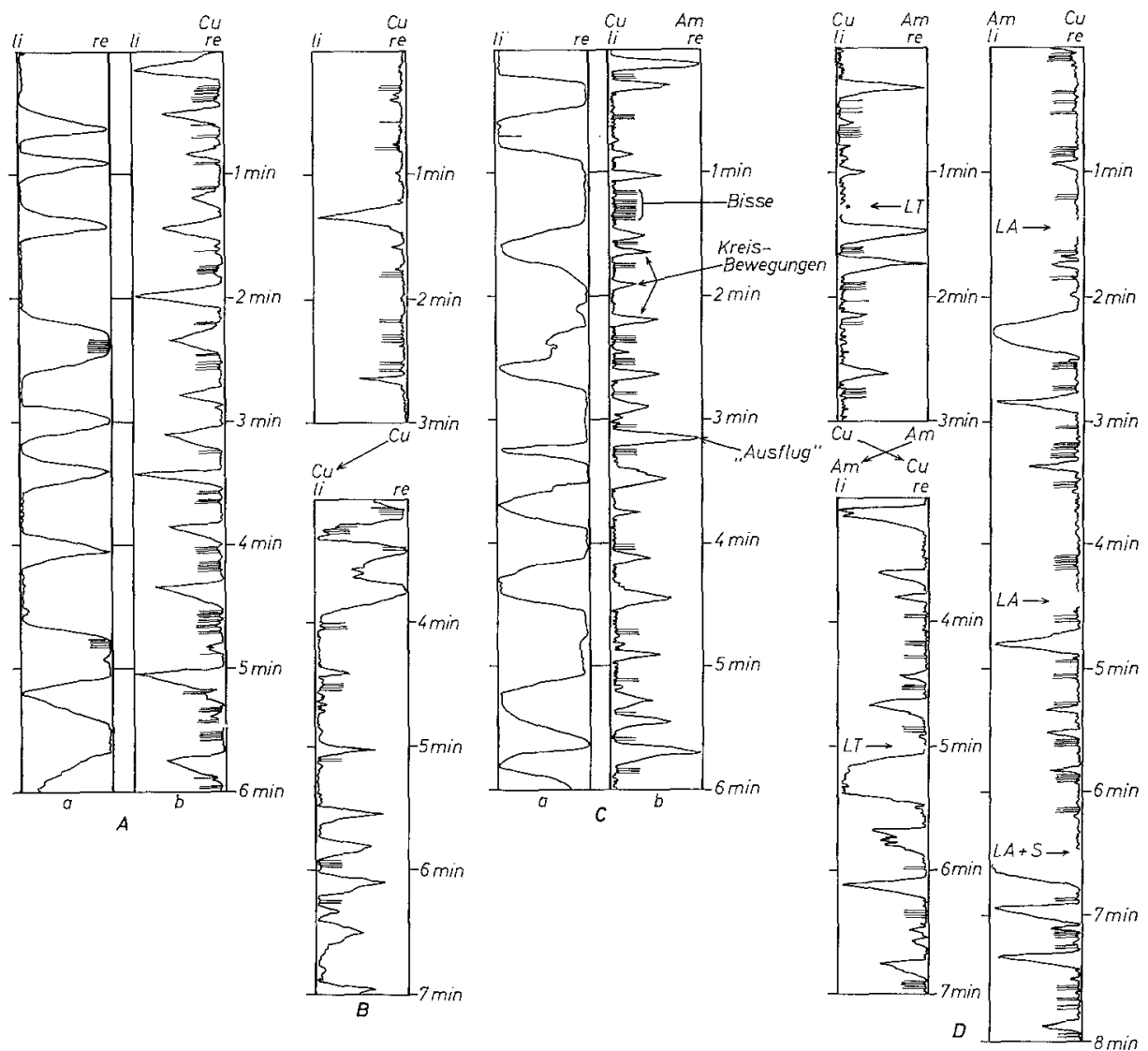

Abb. 2A-D. Direkt registrierte Ausschnitte der Schwimmbewegungen operierter Fische unter verschiedenen Testbedingungen. A Schwimmverhalten und Bisse eines operierten Fisches gegenüber Cumarin zu verschiedenen Zeiten der Lernperiode nach Regeneration. 98. Dressur: a) Kontrolle ohne Geruch: leichte Bevorzugung der linken Seite; b) Test mit Cumarin rechts: deutliche Beantwortung des Geruchs (Verweilen, Bisse, Kreise und Umkehrbewegungen). B Wechsel der ",positiven “ Seite und Neuorientierung im Test mit Cumarin. C Verhalten eines operierten Fisches zu verschiedenen Zeiten der Differenzdressur mit Cumarin (bedingter Reiz) und Amylacetat (konkurrierender Reiz). 141. Dressur (41. Sitzung mit 2 Geruchsstoffen): a) Kontrolle ohne Geruch: leichte Bevorzugung der rechten Seite; b) Test mit Cumarin links und Amylacetat rechts: deutliche Beantwortung des bedingten Reizes mit Verweilen, Bissen, Kreis- und Umkehrbewegungen. D Gleichzeitige Vertauschung der "positiven" (Cumarin) und der ,,negativen" (Amylacetat) Seite während der Testaufzeichnung mit 2 Geruchsstoffen. ,Lockversuch" durch Berührung des Trichters $L T$. Durchgehende Registrierung $(8 \mathrm{~min})$ ohne Seitenwechsel: $L A$, Lockversuch " mit 15-25 ml Amylacetat $4 \times 10^{-3} ; L A+S$, Lockversuch " mit 15-25 ml Amylacetat $4 \times 10^{-3}$; zusätzlich wird versucht, den Fisch mit einem Glasstab auf die negative Seite zu treiben 
Aus diesen Befunden kann gefolgert werden, daß das Lern- und Differenzierungsvermögen operierter Tiere mit regenerierten Fila olfactoria (histologische Befunde s. u.) ebenso gut ist wie das nichtoperierter Tiere: die funktionelle Regeneration der Fila olf. ist in vollem Maße eingetreten.

\section{Spezifische Regeneration nach Durchschneidung der Fila olfactoria; Bulbusexstirpation; Scheinoperation}

Für die weiteren Fragestellungen wurden folgende Versuchsgruppen von je 3 Fischen gebildet:

a) Nach Abschluß einer Differenzdressur (mindestens 20 aufeinanderfolgende Testsitzungen mit deutlichem Lernerfolg) wurden bei 4 Gruppen (vgl. Tabelle 2) die Fila olfactoria durchtrennt.

b) Zusätzlich wurden 4 Kontrollgruppen, die in mehr als 90 Sitzungen auf einen Riechstoff dressiert worden waren, operiert (vgl. Tabelle 2):

Mit zwei Gruppen wurde eine Scheinoperation durchgeführt: Narkose, anschließend Öffnung der Schädelhöhle ohne weiteren operativen Eingriff.

Bei den zwei anderen Gruppen wurden operativ die Bulbi olfactorii abgetrennt und entfernt. Damit sollte eine Regeneration der Verbindung zwischen Mucosa olfactoria und Vorderhirn für längere Zeit unterbunden werden.

Wie die scheinoperierten Kontrollgruppen zeigen (Tabelle 2, Gruppen $5 \mathrm{CK}, 6 \mathrm{AK}$ ), beeinflussen Eingriff und Narkose den Lernerfolg nicht nennenswert, obwohl die Tiere sich 15-25 min in Narkose außerhalb des Wassers befanden. Lediglich die Aktivität und die Beißfreudigkeit der Tiere ist für 1-2 Tage vermindert. Bereits 5 Std nach der Operation beantworten die Fische eine Riechstoffkonzentration von $1-2 \times 10^{-6}$ (g bzw. ml konzentrierter Geruchsstofflösungen auf $1 \mathrm{ml}$ Frischwasser) deutlich; eine Konzentration, auf die bulbus-exstirpierte Fische auch nach vielen Tagen noch nicht reagieren.

Fische mit durchtrennten Fila olfactoria nehmen wenige Stunden nach der Operation Futter auf, reagieren aber, wie Abb. 3 zeigt, auf keinen der in der Dressur verwendeten Riechstoffe in der üblichen Konzentration (Tabelle 2), gleichgültig, ob sie auf einer Seite des Aquariums allein (Generalisationstest) oder auf beiden Seiten des Aquariums (verschiedene Geruchsstoffe, Differenztest) einströmen. Bei ausbleibender Reaktion auf Tubifexextrakt (Futtergeruch) wurde immer eine nichtoperierte Kontrollgruppe mit derselben Konzentration getestet (Abb. 3 and 2C). 1-6 Tage nach der Operation läßt sich lediglich eine leichte Aktivitätssteigerung, die meist nach $1-3$ min Testdauer abklingt, feststellen. Das gilt sowohl für Tiere mit durchtrennten Fila olfactoria als auch für Tiere, denen der Bulbus olfactorius herausgeschnitten wurde 
Regeneration der Fila olfactoria beim Goldfisch

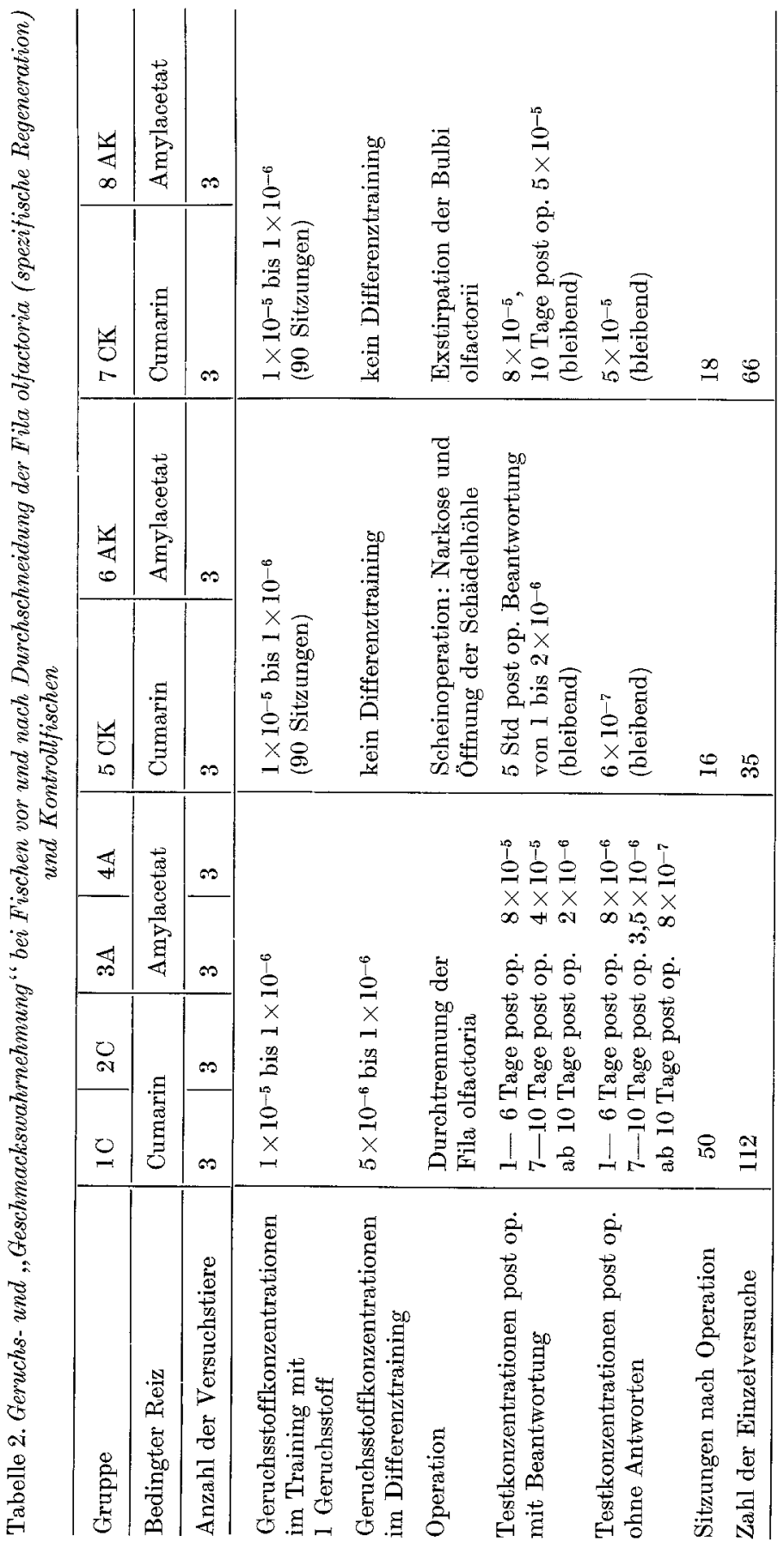



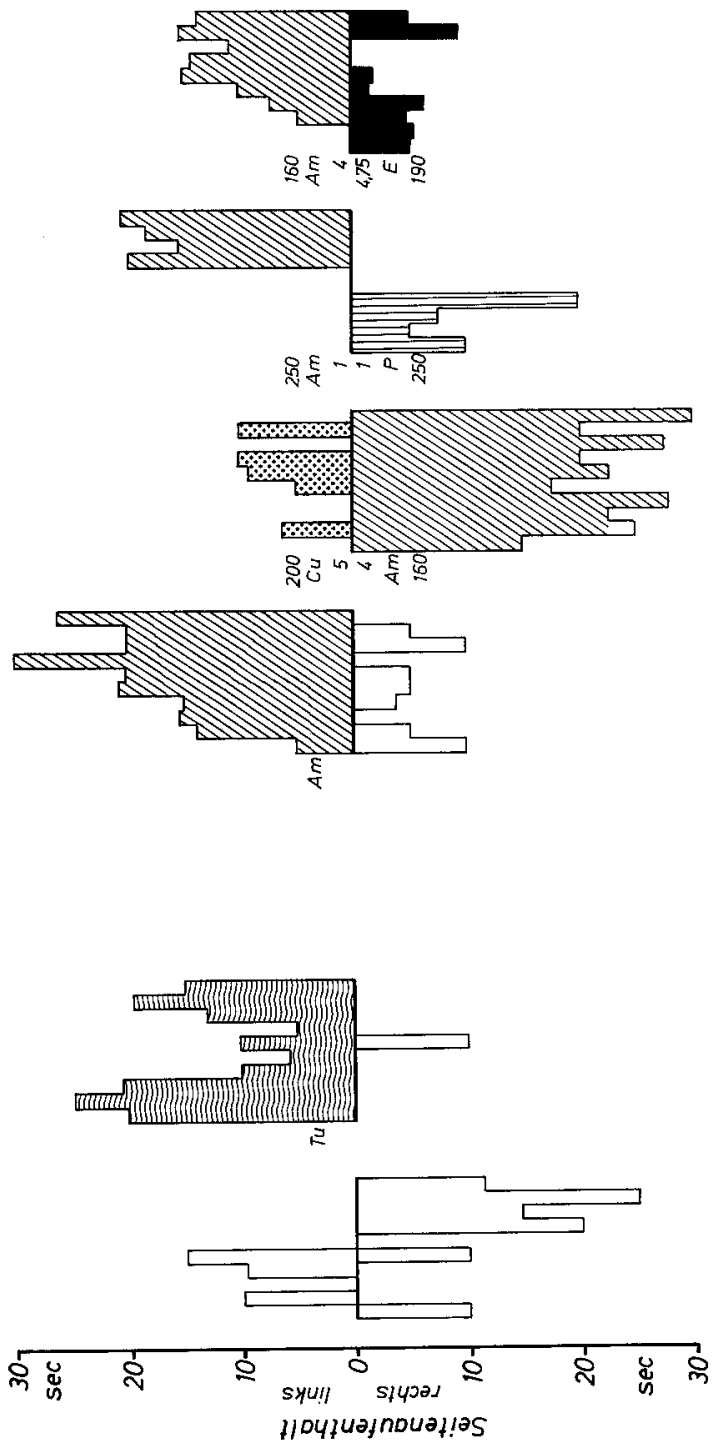

OS.

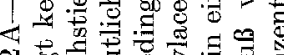
ง.

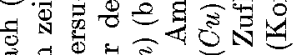

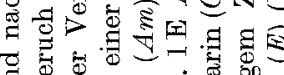

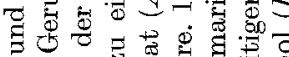

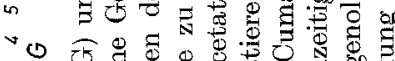
m

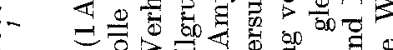

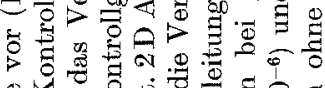
品 8 mL i

$\because$ न ब

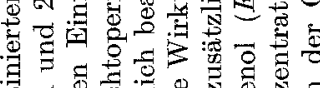
in

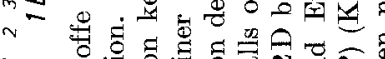

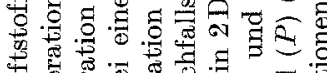

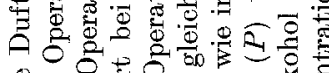
in

iv 검

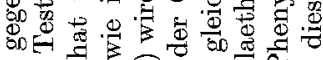
东

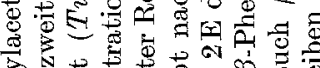
究

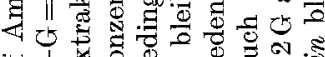
: 元। im iv -

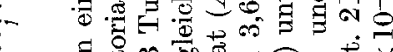
궁

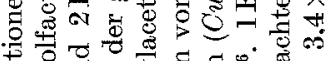

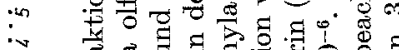
唡

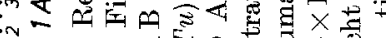

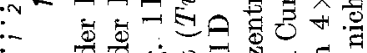



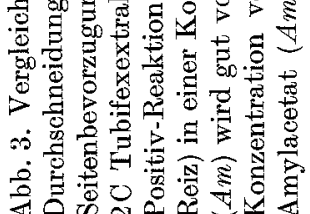



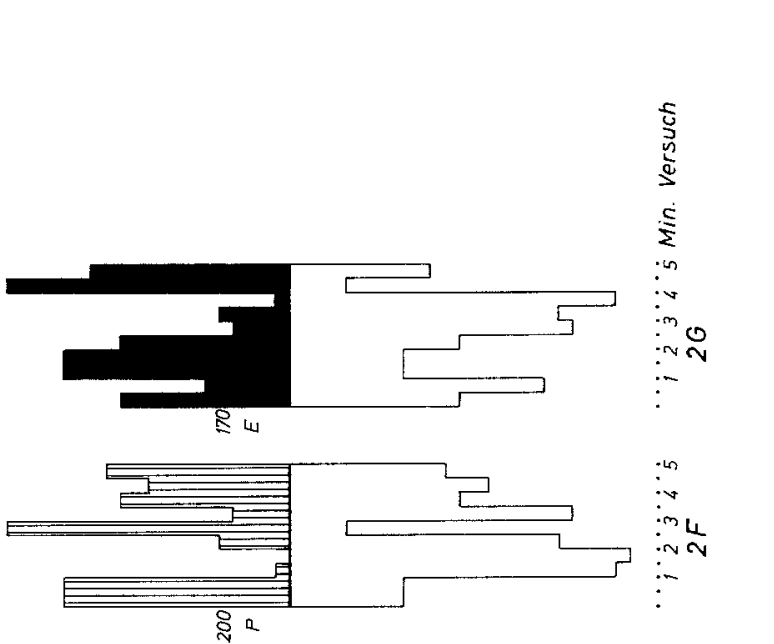

$i^{m} \pi$

$\vdots$

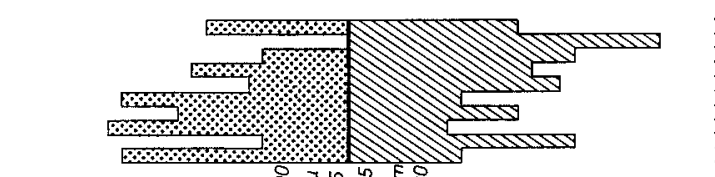

:

im

:v

:-
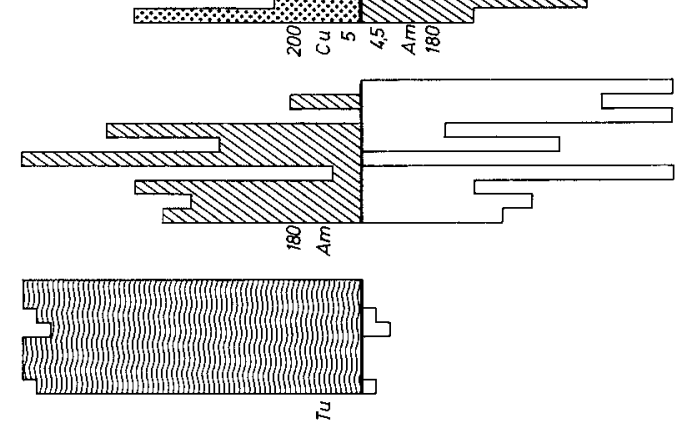

in
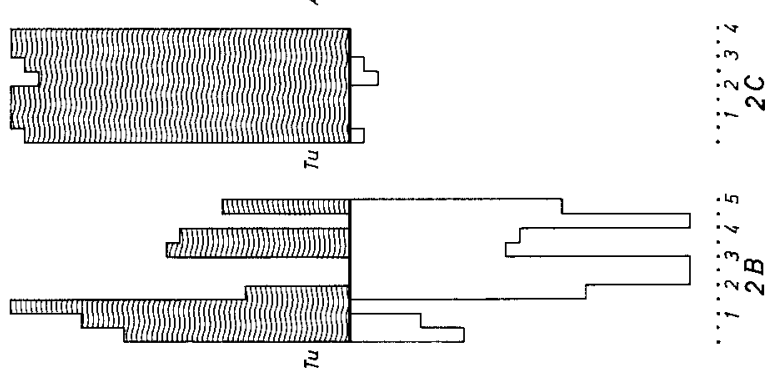

$: 5$

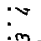

$\lim$

in
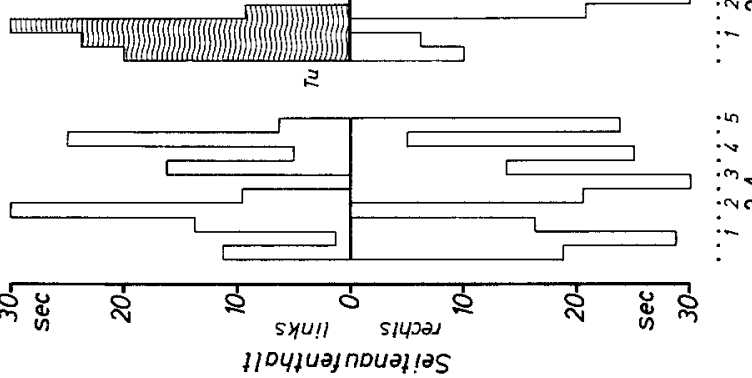

:

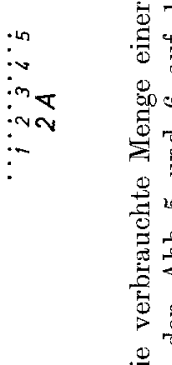

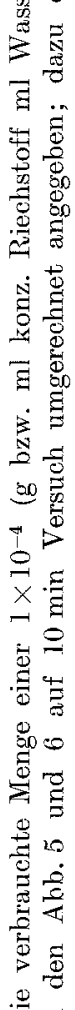

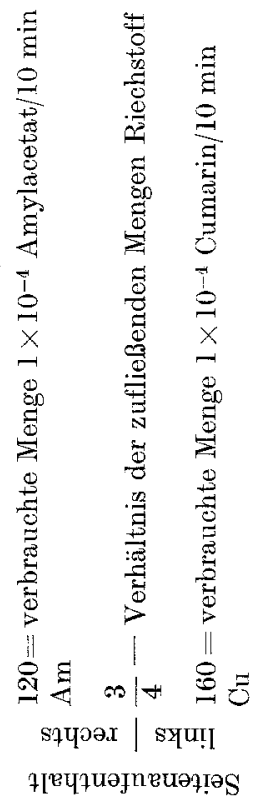



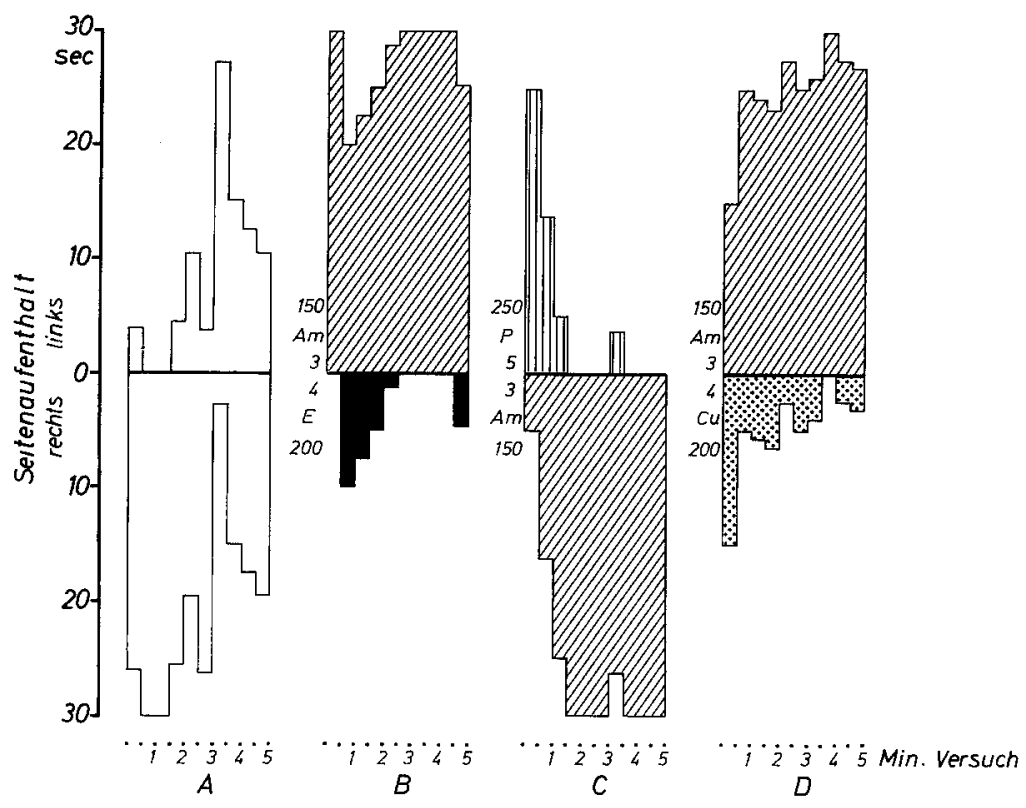

Abb.4A-D. Testversuch nach Wiederherstellung der Riechfähigkeit einer auf Amylacetat trainierten Gruppe (3A). Zehnte Testsitzung nach der Operation (12 Tage post. op.). (Amylacetat als Dressurstoff; Eugenol, $\beta$-Phenylaethylalkohol, Cumarin als zweite Geruchsstoffe). A Kontrolle ohne Geruch mit Bevorzugung der rechten Seite des Aquariums. B Dressurgeruch in einer Konzentration von $3 \times 10^{-6}$ links und ein zweiter Riechstoff (Eugenol) in einer Konzentration von $4 \times 10^{-6}$ rechts: positive Reaktion auf Amylacetat $(\mathrm{Am}) \mathrm{C}$ Amylacetat (Konzentration wie in $\mathrm{B}$ ), jetzt rechts, wird ebenfalls positiv beantwortet, wenn links $\beta$-Phenylaethylalkohol $(P)$ in einer Konzentration von $5 \times 10^{-6}$ einströmt. D Amylacetat links (Konzentration wie in B). Cumarin ( $C u$ ) rechts in einer Konzentration von $4 \times 10^{-6}$. Positive Reaktion auf Amylacetat. Angaben über Verhältnis und Konzentrationen der Geruchsstoffe wie in Abb. 3

(s. Tabelle 2, Gruppe $1 \mathrm{C}$ bis $4 \mathrm{~A}$, Gruppen $7 \mathrm{CK}, 8 \mathrm{AK}$ auch für die folgenden Ergebnisse). Erhöht man jedoch die Konzentration bis nahe $1 \times 10^{-5} \mathrm{~g}$ bzw. ml konzentrierten Geruchstoffes auf $1 \mathrm{ml}$ Wasser (also etwa die 4-8fache Konzentration gegenüber der bei den scheinoperierten Tieren) so reagieren sowohl Versuchstiere mit durchschnittenen Fila olfactoria als auch Bulbus-exstirpierte Tiere vor allem bei Einleitung nur eines Stoffes. Das gilt für alle untersuchten ,,Riechstoffe" sowie für Tubifexextrakt von genügend hoher Konzentration. Bietet man Fischen mit durchtrennten Fila olfactoria einen zweiten Stoff in derartig hoher Konzentration, so wird ihr Verhalten unbestimmt. Offensichtlich handelt es sich dabei um ,Geschmackswirkungen“ der Riech- 
stoffe, die bei Einleitung eines Stoffes allein zur Generalisation führen; werden aber zwei Qualitäten geboten, so können sie wegen der fehlenden geschmacklichen Differenzdressur (die Geruchsdressur erfolgt ja mit niedrigeren als den oben genannten Konzentrationen) nicht unterschieden werden.

7-10 Tage nach der Fila-Durchtrennung (vgl. Tabelle 2) treten bereits vereinzelte Reaktionen auf den bedingten Reiz auf: Es werden wieder Geruchsstoffkonzentrationen wahrgenommen und beantwortet, die deutlich unter der "Geschmacksschwelle" liegen. Diese Reaktionen bleiben vorwiegend auf den bedingten Reiz bezogen, selbst wenn der konkurrierende Riechstoff bei oder leicht über der „,Geschmacksschwelle" liegt. Das Gesamtverhalten in dieser Periode setzt sich vorwiegend aus dem Seitenaufenthalt und Bissen auf der Seite des bedingten Reizes zusammen; die typischen Kreisbewegungen lassen sich noch nicht häufig beobachten.

10-12 Tage nach der Operation kann man auch bei Konzentrationen unter $2 \times 10^{-6}$ deutlich positive Reaktionen, wie sie für die letzten Sitzungen vor der Operation charakteristisch waren (Abb. 3, $1 \mathrm{~A}-\mathrm{G}$ ), beobachten (Abb. 4): Seitenaufenthalt und Beißbewegungen sowie Kreisbewegungen sind in vollem Umfang vorhanden und bleiben auch in längeren Testsitzungen bei stärkerer Konzentration des konkurrierenden Riechstoffes stets auf den bedingten Reiz bezogen, obwohl nach der Operation keine erneate Dressur durchgeführt wurde. Hieraus kann geschlossen werden: 1. Das Gedächtnis für den bedingten Reiz und dessen präoperativ erlernte Qualität bleibt über die Regenerationszeit erhalten. 2. Die Regeneration der Fila olfactoria ist so eingetreten, daß eine ,, sinnvolle Übermittlung“" der Geruchsqualität zum Bulbus olf. gewährleistet wird. Damit ist die ,,spezifische Regeneration" der zuvor erlernten olfaktorischen Leistungen eingetreten. Bei Untersuchungen der ", Geschmackswirkungen " an den Bulbus-exstirpierten Tieren stellt sich heraus, daß die Geschmacksschwellen für einzeln eingeleitete Riechstoffe in den ersten Testsitzungen nach der Operation bei $8 \times 10^{-5}$ liegen, später erniedrigen sie sich auf $6 \times 10^{-5}$. In späteren Sitzungen, 10-20 Tage nach der Operation, erhält man niemals eindeutige Reaktionen unter einer Konzentration von $5 \times 10^{-5}$. Das deutlichste Verhalten (Seitenaufenthalt, Bisse und Kreisbewegungen genau wie bei der Wahrnehmung von Riechstoffen) löste bei diesen Konzentrationen Amylacetat aus; Cumarin und Eugenol wirkten schwächer, waren aber noch gut registrierbar, für $\beta$-Phenyl-aethylalkohol ergab sich selten eine Reaktion unter $1 \times 10^{-7}$. Genauere Untersuchungen über das ,, geschmackliche " Lernen und Differenzierungsvermögen bei der Dressur mit Riechstoffen hoher Konzentration lagen nicht im Interesse dieser Arbeit. 


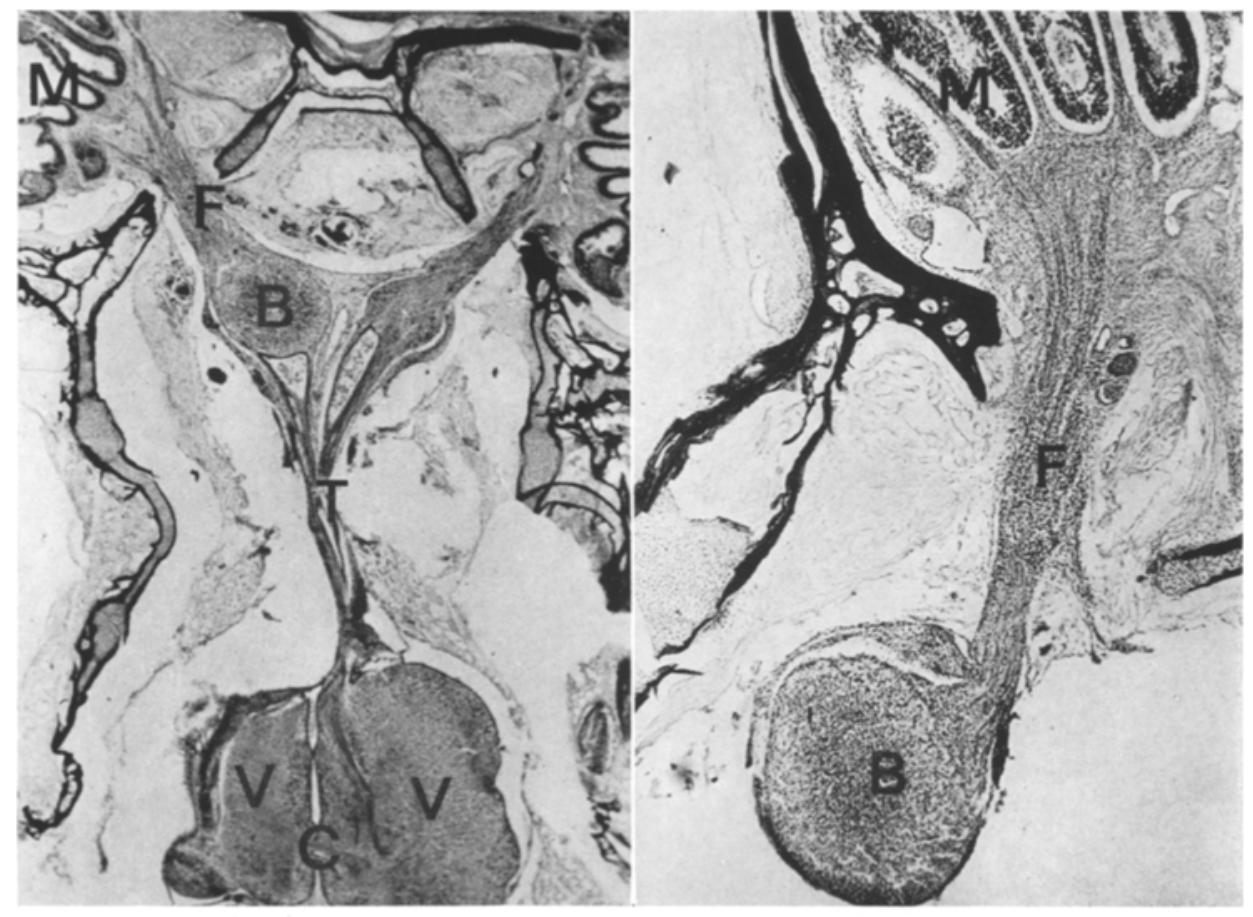

A

B

Abb. 5A-D. Ausschnittvergrößerung aus zwei Fischgehirnen $1 \frac{1}{2}$ Jahre nach Durchschneidung der Fila olfactoria und Kreuzung der Bulbi olfactorii. Schnittrichtung: horizontal längs; Schnittdicke: $16 \mu$. A Übersichtsvergrößerung mit $\mathrm{Mu}$ cosa olfactoria $(M)$, regenerierten Fila olfactoria $(F)$, Bulbus olfactorius $(B)$, Tractus olfactorius $(T)$ mit Kreuzung (1 Tractus nur angeschnitten), Vorderhirnhemisphären $(V)$ und Commissura anterior $(C)$. Vergr. etwa $12 \times$. B Ausschnittvergrößerung von der Mucosa olfactoria $(M)$ bis zum Bulbus olfactorius $(B)$. Deutlich sind die Filamente $(F)$ von der Mucosa bis zum Bulbus olfactorius zu erkennen. Vergr. etwa $35 \times . \mathrm{C}$ und $\mathrm{D}$ abirrende Fasern aus den Fila olfactoria. C Ausschnittvergrößerung von der Mucosa olfactoria $(M)$ bis zum Bulbus olfactorius $(B)$. Ein abirrendes Bündel $(A F)$ zieht aus den Fila olfactoria $(F)$ heraus in Richtung auf den ursprünglich ipsilateralen Bulbus olfactorius. Vergr. etwa $35 \times$. D dasselbe abirrende Bündel wie in C in stärkerer Vergr. etwa $120 \times$

\section{Histologische Regeneration und spezifische Regeneration nach Durchtrennung der Fila olfactoria}

a) Regeneration der Fila olfactoria nach Abschluß der Einzeldressur $\left(1^{1} / 2\right.$ Jahre nach derOperation $)$. Bei diesen Fischen waren bei der Operation neben der Durchtrennung der Fila olfactoria zusätzlich die Bulbi olfactorii von der ipsi- zur contralateralen Seite verlegt worden. Damit war die restlose Durchtrennung der Fila olfactoria sichergestellt, die allerdings 

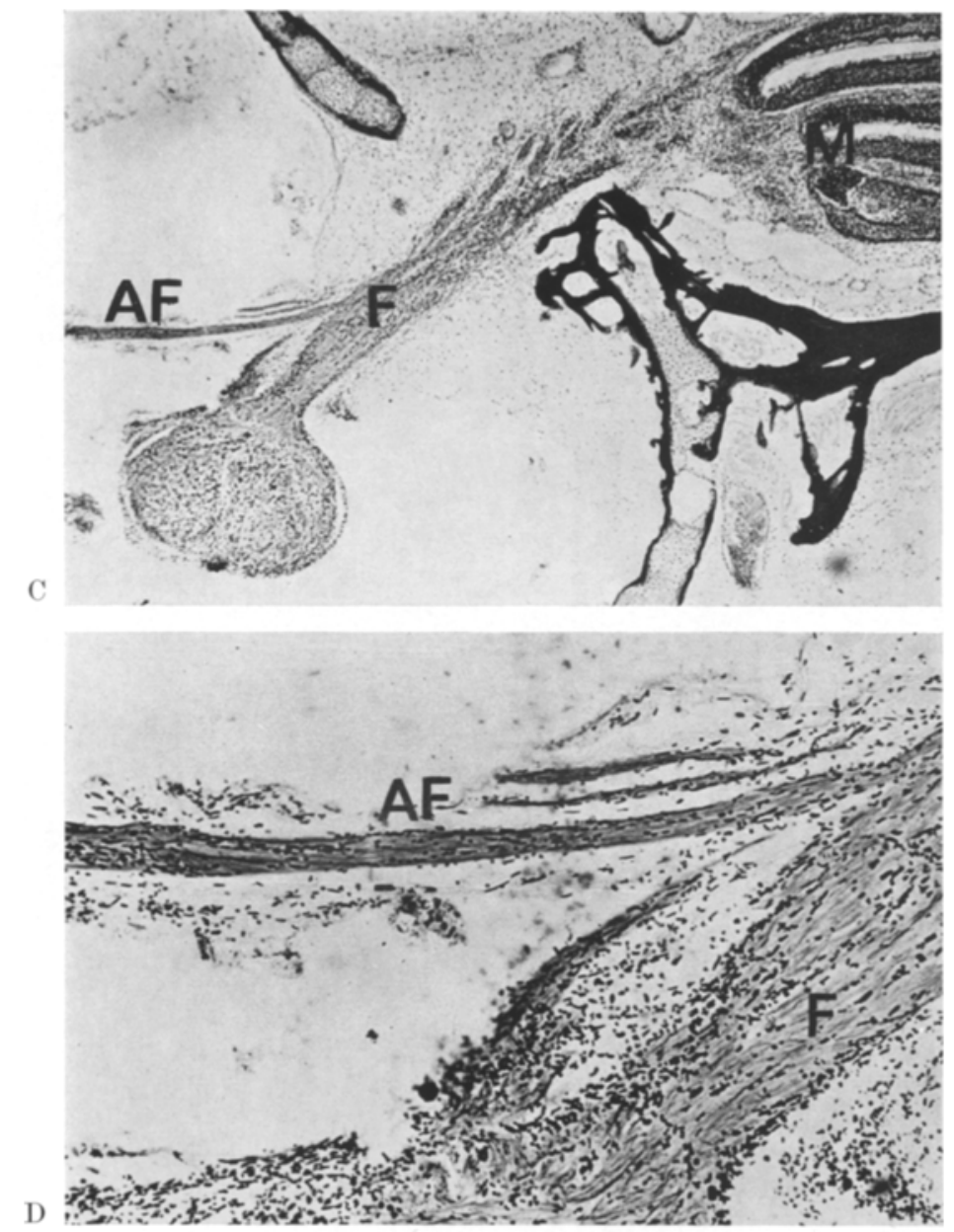

Abb. 5C und D

auch beim Zurückziehen des Bulbus um eine Bulbuslänge gewährleistet ist. Außerdem sollte am olfaktorischen System die bilaterale Gleichwertigkeit der olfaktorischen Strukturen aufgezeigt werden (vgl. Sperry, 1945: Überkreuzung der Sehbahnen). Die Nase ist funktionell monorrhin; für die Übermittlung der Informationen ist es gleichgültig, über welche Stationen (linker oder rechter Bulbus) sie im folgenden Zentrum, dem Vorderhirn, eintreffen. Die histologischen Befunde bestätigen diese Annahme in vollem Maße: Der größte Teil der aus der Mucosa olfactoria ziehenden Fila olfactoria (Abb. 5) hat mit dem ursprünglichen contralateralen Bulbus olfactorius Kontakt aufgenommen und damit die 


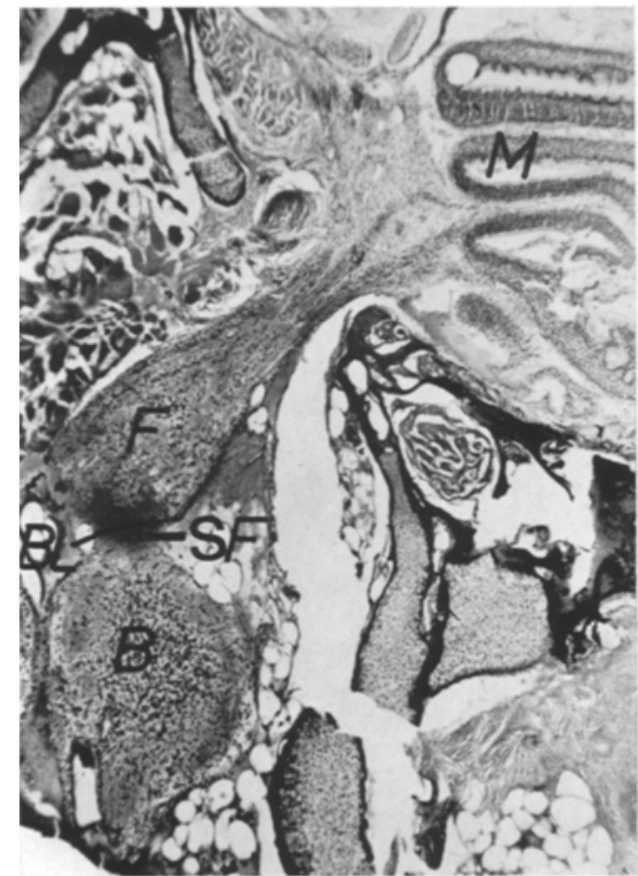

A a

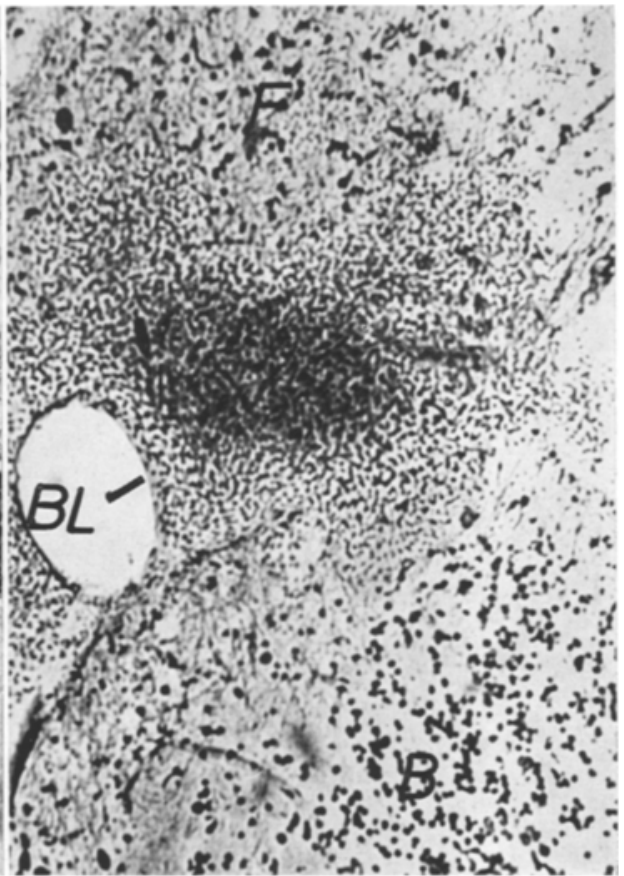

$\mathrm{Ab}$

Abb. 6. A Ausschnittvergrößerungen aus einem Fischgehirn 1 Tag nach Durchschneidung der Fila olfactoria. Schnittrichtung und Schnittdicke wie in Abb. 5 . a Übersicht von der Mucosa olfactoria $(M)$ über die Fila olfactoria $(F)$ mit der Schnittstelle $(S F)$ der Fila und Blutpfropf $(B L)$ in Bulbusnähe $(B)$. Vergr. etwa $35 \times . \mathrm{b}$ die stärkere Vergrößerung der Abb. A zeigt die restlose Durchtrennung der Fila olfactoria. Vergr. etwa $170 \times$. B Ausschnittvergrößerung aus einem Fischhirn 7 Tage nach Durchtrennung der Fila olfactoria. a Úbersicht von der Mucosa olfactoria $(M)$ über die Fila olfactoria $(F)$ mit der Schnittstelle $(S F)$ der Fila und Blutpfropf $(B L)$ in Bulbusnähe $(B)$. Vergr. etwa $35 \times$. b die stärkere Vergrößerung der Abb. a zeigt deutlich „Verdrängungspfade“ durch den Blutpfropf (Pfeile). Vergr. etwa $140 \times$

Verbindung zur contralateralen Vorderhirnhälfte hergestellt (Mucosa-ipsilateral $\rightarrow$ Bulbus-contralateral $\rightarrow$ Vorderhirnhemisphäre-contralateral). Diese regenerierten Bahnen ließen sich bei allen Fischen histologisch eindeutig nachweisen.

Neben diesem Befund ließen sich - ebenfalls bei allen Versuchstieren - auch Faserzüge aus der Mucosa olfactoria verfolgen, die nach dem Austritt aus der Lamina cribriformis des Ethmoids nicht zum contralateralen Bulbus ziehen, sondern - die operativ erzeugte Bulbuskreuzung wieder aufhebend - zum ursprünglich ipsilateralen Bulbus 

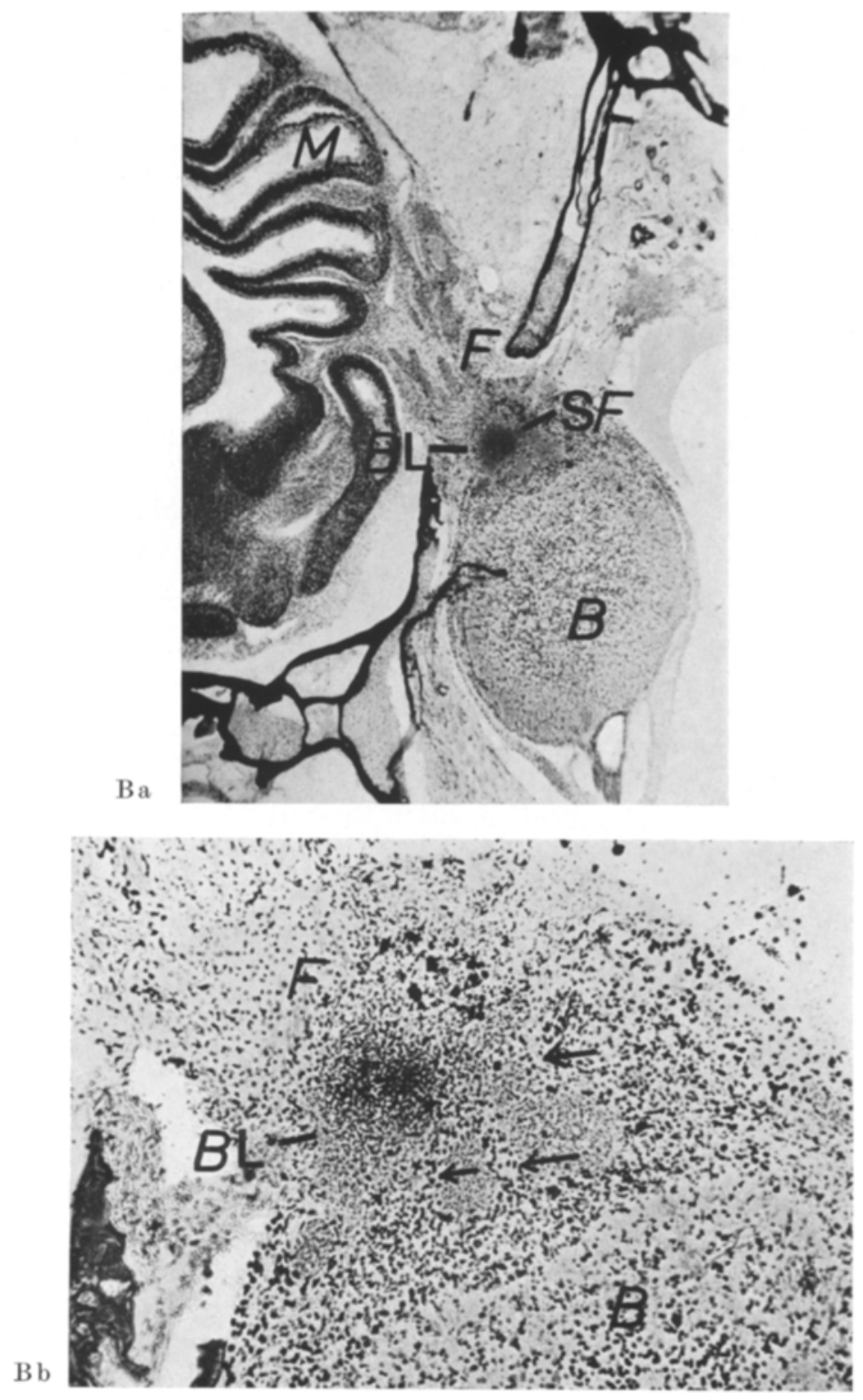

Abb. $6 \mathrm{Ba}$ und $\mathrm{Bb}$

zurückziehen (Abb. 5C). Hier strahlen sie in die Masse der contralateral ziehenden Fila olfactoria aus der gegenüberliegenden Mucosa ein und sind nicht eindeutig weiter zu verfolgen. Es handelt sich bei 
diesen abirrenden Fasern um den wesentlich geringeren Anteil der aus der Mucosa zum Bulbus ziehenden Faserbündel, der größte Teil zieht zum contralateralen Bulbus. Aus diesen Befunden kann geschlossen werden, daß die funktionelle Regeneration (s. o.) nach Durchschneidung der Fila olfactoria durch die morphologische Regeneration der durchschnittenen Bahnen eingetreten ist.

b) Histologische Kontrollserie für die spezifische Regeneration (1,2,4, 7, 16 Tage nach Durchschneidung der Fila alfactoria). Diese Operationen wurden ohne Bulbusüberkreuzungen durchgeführt, um eine möglichst rasche Kontaktaufnahme der Fila mit dem zugehörigen Bulbus olfactorius zu gewährleisten (Verlängerung der Regenerationsstrecke für die Fila olfactoria bei Bulbuskreuzung). Einen Tag nach der Durchschneidung der Fila olfactoria sieht man bei allen Tieren die Ausbildung eines Blutpfropfes, der die Lage der Schnittstelle kennzeichnet. Zusätzlich hält er den durchtrennten Stumpf der aus der Mucosa olfactoria austretenden Fila olfactoria in ihrer ursprünglichen Lage zum Bulbus olfactorius fest (Abb.6A, a, b) und dürfte damit die Regenerationsrichtung im Sinne einer Leitstruktur beeinflussen.

7 Tage nach der Operation lassen sich bereits einige im Mikroskop hell erscheinende Bahnen erkennen, die den Blutpfropf in Richtung: auf den Bulbus olfactorius durchdringen (Abb. 6B, a, b). Es lassen sich in diesen Bahnen größere Zellelemente feststellen, die sich lichtmikroskopisch bei stärkster Vergrößerung nicht von den Schwannschen Zellen der aus der Mucosa ziehenden Fila olfactoria unterscheiden. Wir möchten obwohl Einzelfasern nicht eindeutig zu erkennen sind (Einzelfasern von $0,2 \mu$ Dicke sind lichtmikroskopisch in diesen Präparaten nicht aufzulösen), annehmen, daß es sich hier bereits um Fasern oder kleinere Faserbündel handelt, die in ihrem Regenerationsgang wieder in die Nähe des Bulbus olfactorius gelangt sind oder bereits mit ihm Kontakt aufgenommen haben. Unterstïtzt wird diese Vermutung durch die Verhaltensbefunde.

10-12 Tage nach der Operation, d. h. etwa zum Zeitpunkt des Wiedererscheinens positiver Reaktion (s. o.), erkennt man bei Tieren aus den Dressurserien deutlich Faserverbindungen, die sich durchgehend von der Mucosa olfactoria bis zum Bulbus olfactorius verfolgen lassen (Abb. $7 \mathrm{~A}$ und B). Die Schnittstelle, an der die Fila olfactoria durchtrennt waren, ist auf vielen Schnitten noch deutlich zu sehen: der Blutpfropf ist fast völlig verschwunden.

Damit ist auch histologisch die Grundlage für die spezifisch-funktionelle Regeneration auf Geruchsstoffe dressierter Tiere nachgewiesen: der Eintritt der histologischen Regeneration entspricht dem Zeitpunkt, zu dem auch die Riechfähigkeit wiederhergestellt ist. 


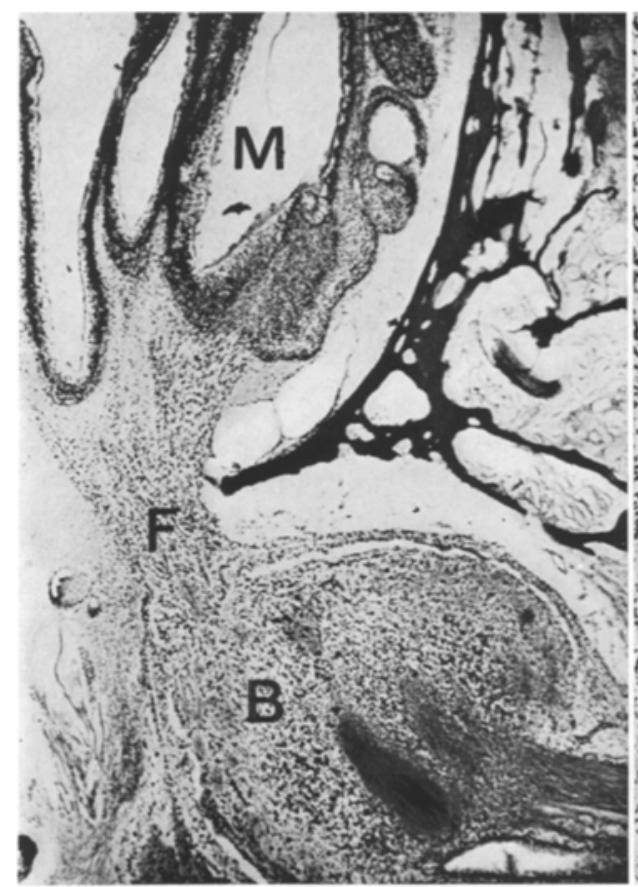

A

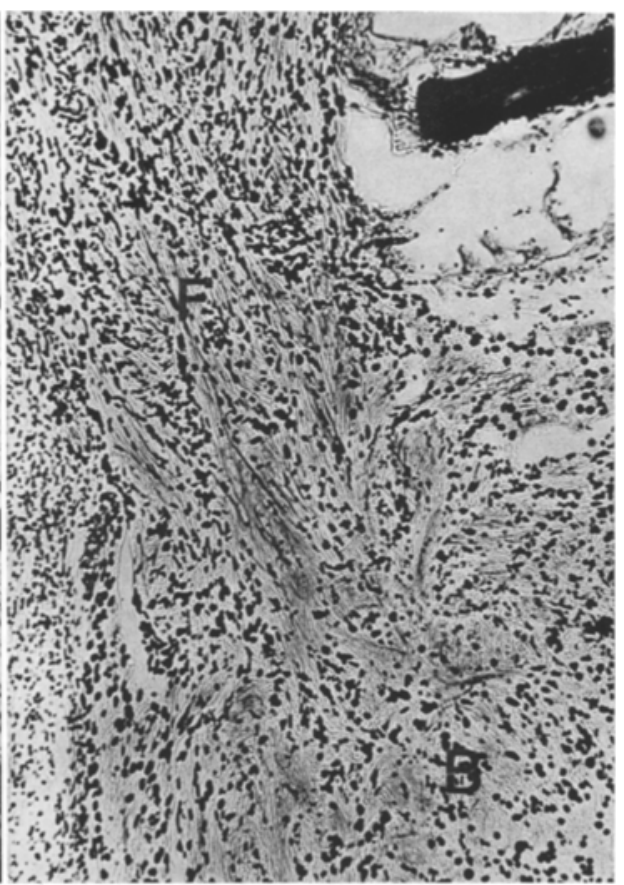

B

Abb. $7 \mathrm{~A}$ und B. Aussehnittvergrößerung aus einem Fischgehirn 12 Tage nach Durchtrennung der Fila olfactoria (Fisch aus der Gruppe $3 \mathrm{~A}$, s. Abb. 4. Schnittrichtung und Schnittdicke wie in Abb. 5). A Übersicht von der Mucosa olfactoria $(M)$ über die Fila olfactoria $(F)$ bis zum Bulbus olfactorius $(B)$. Vergr. etwa $45 \times$. B die stärkere Vergrößerung der Abb. A zeigt die restlose Verdrängung des Blutpfropfs and Faserbündel, die in den Bulbus olfactorius einstrahlen. Vergr. etwa $170 \times$. Nähere Erläuterungen im Text

\section{Diskussion}

Als spezifisch im strengeren Sinne bezeichnen wir eine Regeneration dann, wenn die vorher in der Dressur gebahnten Verbindungen wieder funktionstüchtig werden, d. h., wenn die Tiere die erlernte Aufgabe nach der Regeneration wieder beherrschen; funktionell kann man eine Regeneration nennen, die es dem Fisch ermöglicht, wieder die gleichen dem jeweiligen System zukommenden Aufgaben, in erster Linie angeborene Leistungen, zu vollbringen. Der Nachweis einer histologischen Regeneration allein gibt nur Auskunft über das Vorhandensein von Strukturen, jedoch noch nicht über ihre Funktion.

Eine vollständige funktionelle und histologische Regeneration ließ sich für die Fila olfactoria nachweisen. Die regenerierten Strukturen 
und Funktionen unterscheiden sich nicht von normalen. In diesen Operationen wurden zusätzlich die ipsilateralen Bulbi olfactorii zur contralateralen Seite gebracht; der größte Teil der Fila olfactoria regenerierte zum angebotenen contralateralen Bulbus. Diese Befunde decken sich mit den von Sperry u. Mitarb. durchgeführten Untersuchungen am optischen System. Die abirrenden Faserbündel, welche zum ursprünglichen ipsilateralen Bulbus olfactorius ziehen, unterscheiden sich anatomisch nicht von den oben beschriebenen. Über ihre Funktion kann, da elektrophysiologische Untersuchungen fehlten, vorerst nichts ausgesagt werden.

Die funktionell-spezifische Regeneration, die von Sperry et al. für das optische System bewiesen wurde, tritt nach Durchschneidung der Fila olfactoria in der erstaunlich kurzen Zeit von 10-14 Tagen auf. Etwa ebensolange dauert die histologisch nachweisbare Wiederverheilung der durchtrennten Bahnen. Aus diesem Befund ist zu folgern, daß die „,Behaltezeit“ für das Gelernte erheblich länger als die „,Regenerationszeit" sein dürfte. Vergessenstests lagen jedoch außerhalb des Rahmens dieser Arbeit. Der Regenerationsgang ließ sich in einer Serie für die spezifische Regeneration nach Durchtrennung der Fila olfactoria histologisch verfolgen. Ähnlich wie von Kirsche (1950) für das Rückenmark beschrieben, konnten wir nach der Operation als erstes Anzeichen für die Regeneration ein Durchdringen des Narbengewebes (Blutpfropf) durch aussprossende Faserzüge feststellen, wobei sich in unserem Fall die Einzelfasern $(0,2 \mu \mathrm{im}$ Durchmesser $)$ lichtmikroskopisch nicht darstellen ließen. Etwa gleichzeitig mit der funktionell-spezifischen Regeneration ließ sich auch der Wiedereintritt von Fasern über die ursprüngliche Schnittstelle der Fila olfactoria hinweg bis zum Bulbus olfactorius verfolgen.

Anders als im optischen System (Sperry u. Mitarb.) konnte im olfaktorischen System eine Punkt-zu-Punkt-Regeneration der Mucosa zum Bulbus olfactorius oder vom Bulbus olfactorius zum Vorderhirn bisher nicht sicher beschrieben werden. Sheldon (1912) diskutiert zwar eine solche Projektion der Mucosa zum Bulbus (laterale Mucosa-Bezirke zu lateralen Bulbusteilen), doch fehlen hier bisher ebenso wie hinsichtlich der Projektion der aus dem Bulbus in das Vorderhirn einstrahlenden Tractus-Fasern, die vorwiegend zu bestimmten Kerngebieten ziehen sollen, eingehende elektrophysiologische Untersuchungen.

Die Kontrollversuche mit Fischen, denen nach Ausbildung des bedingten Reflexes die Bulbi olfactorii entfernt worden waren, legen es nahe, auch an die Mitbeteiligung des Geschmackssinnes zu denken. Möglicherweise spielte er auch bei ähnlichen Untersuchungen anderer Autoren mit: Sowohl Cumarin (Strieck, 1942: Elritze) als auch Eugenol und $\beta$-Phenylaethylalkohol (Neurath, 1949: Elritze) wie weiterhin 
Amylacetat (Sanders, 1940: Goldfisch) und Futter (Parker, 1910) sollen als Riechstoffe, nicht aber geschmacklich wahrgenommen werden, obwohl z. B. Cumarin für menschliches Empfinden einen ausgesprochen bitteren Beigeschmack hat. Diese Behauptungen sind schwer zu halten angesichts unserer positiven Tests mit diesen Stoffen nach totaler Bulbusexstirpation. Es zeigte sich ganz eindeutig - vor allem für Amylacetat ab $5 \times 10^{-5}$ - eine positive, bleibende Reaktion, deren Verhaltenskomponenten sich nicht von den typischen Geruchsreaktionen unterschieden. Das Gleiche gilt, allerdings bei etwas höheren Konzentrationen, für Cumarin und Eugenol. Die schwächste und häufig nicht deutliche Reaktion (ab $1 \times 10^{-4}$ ) konnten wir bei $\beta$-Phenylaethylalkohol feststellen. Auch stark konzentrierter Tubifexextrakt führte bei bulbus-exstirpierten Fischen zu einer Bevorzugung der Seite des Aquariums, auf der er eingeleitet wurde. Diese Befunde mögen durch die straffreie Versuchsanordnung begünstigt sein, denn wenn die Versuchstiere mit Strafe ("shock avoidance training") davon abgehalten werden, auf irgendeinen anderen als ausschließlich den bedingten Reiz zu antworten, dann dürfte bei Ausschaltung des Geruchssinnes nach einer Differenzdressur unterhalb der Geschmacksschwelle auch kaum eine Reaktion (Generalisation) selbst auf höher konzentrierte Riechstoffe und damit auf deren Geschmackskomponenten zu erwarten sein.

Ob sich nach Ausschaltung des Bulbus olfactorius mit den oben beschriebenen Riechstoffen bei genügend starker Konzentration auch eine geschmackliche Differenzdressur durchführen läßt, soll in einer späteren Arbeit untersucht werden.

Bei den kritischen Geruchstests an intakten und operierten Tieren lagen jedoch die Konzentrationen der Duftstoffe stets unterhalb der Geschmacksschwellen, außer bei einigen Sitzungen im Gruppentraining für die spezifische Regeneration, oder gelegentlich absichtlich im Test nach der Operation. Bei diesen niedrigen Konzentrationen ist an der echt olfaktorischen Natur der beobachteten sensorischen Leistungen nicht zu zweifeln.

\section{Literatur}

Arora, H. L., Sperry, R. W.: Color discrimination after nerve regeneration in the fish (Astonotus ocellatus). Develop. Biol. 7, 234-243 (1963).

Attardi, D. G., Sperry, R. W.: Preferential selection of central pathways by regenerating optic fibres. Exp. Neurol. 7, 46 (1963).

Kirsche, W.: Die regenerativen Vorgänge am Rückenmark erwachsener Teleostier nach operativer Kontinuitätstrennung. Z. mikr.-anat. Forsch. 56, 190-265 (1950).

Neurath, H.: Utber die Leistung des Geruchssinnes bei Elritzen. Z. vergl. Physiol. $31,609-626$ (1949).

Parker, G. H.: The olfactory reactions in fishes. J. exp. Zool. 8, 535-542 (1910).

Sanders, F. K.: Second order olfactory and visual learning in the optic tectum of the goldfish. J. exp. Biol. 17, 416--434 (1940).

7 Z. vergl. Physiologie, Bd. 69 
Sheldon, R. E.: The olfactory tracts and centers in teleosts. J. comp. Neurol. 22, No $3,177-339$ (1912).

Sperry, R. W.: Restoration of vision after crossing of optic nerves and after contralateral transplantation of eye. J. Neurophysiol. 8, 15-28 (1945).

- Regulative factors in the orderly growth of neural circuits. Growth 10, 63-87 (1951).

Strieck, F.: Untersuchungen über den Geruchs- und Geschmackssinn der Elritze (Phoxinus laevis). Z. vergl. Physiol. 2, 122-154 (1942).

Zippel, H. P., Westerman, R. A.: Geruchsdifferenzierungsvermögen der Karausche (Carassius carassius) nach funktioneller und histologischer Regeneration des Tractus olfactorius und der Commissura anterior. Z. vergl. Physiol. 69, 38-53 (1970).

- Verhaltenskomponenten und Differenzierungsvermögen in der straffreien Geruchsdressur beim Goldfisch (Carassius auratus). Z. vergl. Physiol. 69, 54-78 (1970).

Dr. H. P. Zippel

Physiologisches Institut der Universität

Lehrstuhl II

D-3400Göttingen,Humboldtallee 7

Dr. R. A. Westerman

Monash University

Clayton, Victoria, Australia
Dr. R. v. Baumgarten

The University of Michigan

Ann Arbor, Mich. 48104, U.S.A. 